\title{
Psychosocial therapy for Parkinson's-related dementia: intervention development
}

This article was published in the following Dove Press journal:

Clinical Interventions in Aging

20 October 2017

Number of times this article has been viewed

\author{
Sheree A McCormick ${ }^{1,2, *}$ \\ Kathryn R McDonald ${ }^{1,2, *}$ \\ Sabina Vatter ${ }^{1-3}$ \\ Vasiliki Orgeta ${ }^{4}$ \\ Ellen Poliakoff',2 \\ Sarah J Smith ${ }^{5}$ \\ Iracema Leroi ${ }^{1-3}$ \\ 'Division of Neuroscience and \\ Experimental Psychology, University \\ of Manchester, Manchester, UK; \\ ${ }^{2}$ Manchester Academic Health Science \\ Center (MAHSC), Manchester, UK; \\ ${ }^{3}$ Greater Manchester Mental Health \\ Foundation Trust, Manchester, UK; \\ ${ }^{4}$ Division of Psychiatry, University \\ College London, London, UK; ${ }^{5}$ Faculty \\ of Health Studies, University of \\ Bradford, Bradford, UK \\ *These authors contributed equally \\ to this work
}

\begin{abstract}
Background: Group-based psychosocial therapy, such as group Cognitive Stimulation Therapy, improves cognition and quality of life in people living with dementia. Neuropsychiatric symptoms and restricted mobility are common complications for people with Parkinson's-related dementia (PRD) and may limit access to, and participation in, group activities. This study describes the development of a condition-specific, home-based psychosocial therapy for people with PRD ready to be trialled in a clinical population.
\end{abstract}

Methods: By means of a multistage process, a draft therapy manual was developed in an iterative manner through collaboration with medical experts, researchers and Patient and Public Involvement (PPI) representatives. In stage 1, an extensive literature search of psychosocial therapies for dementia with potential relevance for Parkinson's disease (PD) was undertaken to select a candidate therapy for adaptation. In stage 2, qualitative feedback from stakeholders and intelligence regarding existing nonpharmacological therapies for cognitive impairment in PD was combined to produce a prototype therapy manual. In stage 3 , the manual was field tested in: 1) a home-setting using a 25 -item assessment tool; and 2) at a local PD support group with PPI representatives. Based on the feedback from this phase, final design modifications were implemented and a draft therapy manual produced.

Results: The manual was developed in an iterative manner. Interview and focus group transcripts identified three enduring themes: manual form and content, therapy acceptability by people with PRD, and companion guidance and support. Major adaptations included: removal of discrete levels of task complexity, removal of images that were potentially hallucinogenic or lacked clarity, and updating of the content.

Conclusion: We have successfully developed a Cognitive Stimulation Therapy-based psychosocial therapy specifically adapted for people with PRD. The therapy is ready to trial in a pilot randomized controlled study.

Keywords: Parkinson's disease, cognitive stimulation, MRC framework, intervention development

\section{Introduction}

England's National Institute for Health and Clinical Excellence guidelines ${ }^{1}$ recommend that people with dementia have timely access to a psychosocial therapy, such as group Cognitive Stimulation Therapy (CST). Therapies such as CST may be appropriate for the less common types of dementia; however, they often do not meet the more complex needs (ie, restricted mobility, development of an inferiority complex within a group and neuropsychiatric symptoms) of people with dementia in the context of movement disorders.

Individuals with Parkinson's disease (PD) can experience a wide range of motor and non-motor symptoms that present challenges in undertaking day-to-day activities. ${ }^{2-4}$ 
Approximately $30 \%$ of people with early, unmedicated PD report problems with attention and memory. ${ }^{5}$ A quarter of people with PD will develop mild cognitive impairment (PD-MCI) and are at increased risk of developing dementia. ${ }^{6}$ The likelihood is that over $80 \%$ of people with PD will develop dementia after 20 years. ${ }^{7}$ People who have dementia with Lewy bodies (DLB) present with similar cognitive and motor symptoms to those with PD and dementia (PDD $)^{8}$ and face similar challenges in accessing currently available psychosocial treatment. Thus, there is a clinical need to develop and evaluate psychosocial support interventions for people with Parkinson's-related dementia (PRD).

The Medical Research Council (MRC) guidelines for developing and evaluating complex interventions ${ }^{9}$ recommend that complex interventions are developed systematically using the best available evidence and supported by an appropriate theoretical framework. Despite these guidelines, early findings from an ongoing Cochrane review of trials of cognitive training (a technique focusing on the repetition of cognitive exercises that may strengthen impaired cognitive processes) for people with PD and cognitive impairment suggests that relatively few studies meet these criteria. ${ }^{10}$ The guidelines also recommend that interventions be developed to the point where they can reasonably be expected to have a worthwhile effect. One approach to following these guidelines is to modify an intervention that already has a robust theoretical, empirical and clinical rationale and an emerging evidence base of efficacy. The added benefit of this approach is that the development time can be reduced and the intervention is more likely to be scalable and sustainable.

This paper describes the methods and outcomes relating to the adaptation of a psychosocial therapy for people with PRD and their companions. To meet the needs and challenges of this specific clinical population, we adopted a userdriven development framework including principles such as: active user involvement, iterative design, prototyping, evaluations with real users and usability considerations (Figure 1). ${ }^{11}$ Given the increased levels of caregiver burden in this population, the acceptability and tolerability of the therapy by companions (historically referred to as carers or caregivers) was also carefully considered. The effectiveness of the adapted therapy will be subsequently examined in an innovative feasibility and efficacy trial following the MRC's clinical guidelines. ${ }^{9}$

\section{Aims and objectives}

The aim of this study was to develop a comprehensive psychosocial therapy specifically adapted for people with

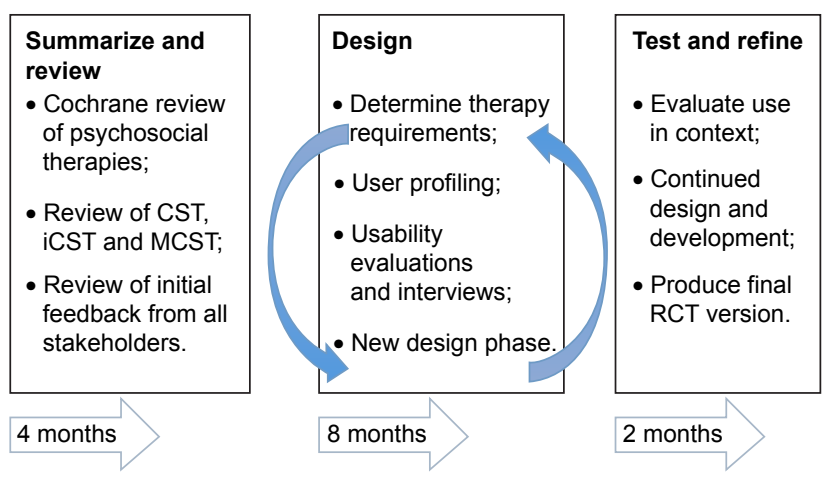

Figure I The user-driven development framework adopted during the development of CST adapted for people with Parkinson's-related dementias (CST-PD).

Abbreviations: CST, Cognitive Stimulation Therapy; iCST, individual Cognitive Stimulation Therapy; MCST, Maintenance Cognitive Stimulation Therapy; PD, Parkinson's disease; RCT, randomized controlled trial.

PRD and their companions. In this context, people with PRD comprise those who have PDD, PD-MCI or DLB. The specific objectives were:

- To conduct an extensive literature search to: 1) summarize and review the existing evidence of psychosocial therapies for cognitive impairment and dementia related to PD; 2) develop a theoretical understanding of the likely processes of change; and 3) select a candidate therapy for adaptation (stage 1; summarize and review).

- Identify aspects of the candidate therapy that need to be adapted for people with PRD: Combine feedback from consultations with Patient and Public Involvement (PPI) representatives, clinical experts and intelligence regarding existing nonpharmacological therapies for cognitive impairment in PD to identify an initial set of adaptations required to produce the prototype therapy manual prior to field testing (stage 2; design).

- Evaluate the design against stakeholder requirements: Field test the prototype therapy manual to: 1) obtain feedback on the usability of the adapted therapy delivered by a companion in a home-setting and 2) obtain feedback from PPI representatives and clinical experts (stage 3; test and refine).

\section{Stage I - summarize and review (existing research)}

To summarize and review the existing evidence of psychosocial therapies for cognitive impairment and dementia related to $\mathrm{PD}$, we conducted a scoping exercise followed by a systematic review of seven studies (the number of studies reviewed is an indication of the paucity of high-quality research in this area). Scoping exercises do not evaluate the quality of the evidence reviewed; however, they can provide 
a narrative integration of evidence when the research area is complex and has not been comprehensively reviewed before. ${ }^{12}$ We considered studies that recruited people with PRD and: 1) reported a comparison between a psychosocial intervention (cognitive-related) and a control group; 2) reported a comparison between pre- and posttest; and 3) reported the experiences of people with dementia participating in a psychosocial intervention. To map rapidly the key concepts underpinning effective cognitive stimulation in this clinical population, we synthesized knowledge from an ongoing, larger systematic review study. ${ }^{10}$ Throughout the review process, stakeholders (clinicians and PPI representatives) were consulted to provide insight on what the literature failed to highlight. The process was iterative, rather than linear, and involved cycling between early findings and new insights. The findings from the scoping and systematic review are now discussed.

Early psychosocial therapies for people with dementia, such as Reality Orientation ${ }^{13}$ and Reminiscence Therapy, ${ }^{14,15}$ evolved in parallel with the aim of reducing disorientation and improving well-being. Reality Orientation involves the use of supporting aids to establish time, season, location and other current or basic personal information, but has been criticized for being rigid and confrontational, with doubts being raised about the therapy's capability for demonstrating any clinically significant improvement. ${ }^{16}$ Supported by the use of memory aids (photographs, books or objects), Reminiscence Therapy involves discussing past experiences and events, but has also been found to have considerable shortcomings. A recent randomized, controlled trial of Reminiscence Therapy with people with dementia demonstrated no clinical benefit compared to "treatment as usual", and anxiety levels had increased in companions. ${ }^{17}$ Continued use of both of these early psychosocial therapies has been cautioned by the American Psychiatric Association. ${ }^{18}$

More recently, use of CST has gained momentum and is recommended by a large number of National Health Service trusts in the UK. The principle behind CST supports the engagement in cognitive and social activities to enhance cognitive functioning and quality of life of people with dementia. The therapy was developed by systematically selecting the aspects of the early psychosocial therapies that were beneficial and building on these techniques by incorporating the discussion of new ideas and thoughts into various cognitively stimulating activities and topics. A randomized, controlled trial of the CST demonstrated improvements in quality of life and cognitive functioning. ${ }^{19}$ Economic analysis showed CST was cost-effective ${ }^{20}$ and that the results for cognition compared favorably with trials of cholinesterase inhibitors for Alzheimer's disease. Since these early studies, the evidence base supporting the use of CST to improve outcomes for people with dementia has continued to grow. ${ }^{19}$

Following research on the effects of group CST, recent research has reported significant effects (quality of life and cognition) for people attending maintenance CST sessions (a follow-on therapy after group CST) $)^{21,22}$ and, to a lesser extent, individual CST (iCST). ${ }^{19} \mathrm{iCST}$ is delivered individually to a person with dementia by a friend or a family member. A recent randomized, controlled trial of iCST showed that iCST did not result in improved cognition or quality of life for the person living with dementia, however, participating in iCST did enhance the quality of the caregiving relationship and caregivers' quality of life. ${ }^{23,24}$ These findings are relevant here, as companions of people with PRD are reported to experience burden and a lower quality of life due to the responsibility of supporting the complex physical and cognitive needs of people with PRD. ${ }^{25} \mathrm{We}$ are aware of only one exploratory study ${ }^{26}$ that has assessed the effectiveness of home-based CST in people with PRD using neuropsychological assessment and molecular analysis. The authors reported that scores on the Montreal Cognitive Assessment, ${ }^{27}$ Parkinson's Disease Questionnaire-39 ${ }^{28}$ and Quality of LifeAlzheimer's Disease ${ }^{29}$ significantly improved following a 7-week period of CST delivered by a family member. In addition, levels of serum brain-derived neurotrophic factor (BDNF; a neuroprotectant that colocalizes with dopaminergic neurons) significantly improved at posttest. The sample size in this study was small and there was no control group; however, the ability to demonstrate positive change in a highly objective biomarker is encouraging.

Given the benefits offered by CST, maintenance CST, iCST and a recent Cochrane Review recommending systematic evaluation of different modalities of CST, including real-life settings, ${ }^{16}$ the home-based companion-delivered iCST was selected as the candidate therapy for adaptation. The modified version is henceforth referred to as CST-PD.

\section{Process of change}

In accordance with MRC guidance for developing and evaluating complex interventions, ${ }^{9}$ a good theoretical understanding of how the intervention effects change is required in order to identify and strengthen areas of weakness within the intervention. ${ }^{30}$ Prior to the design and prototyping stage, a literature scoping exercise was performed to identify the potential processes of change. At a fundamental level, for the people with $\mathrm{PRD}$, the therapy may result in neurobiologic or 
psychosocial changes or a combination of the two. Recent evidence supports the idea that nonpharmacologic therapies exert action on neurobiologic processes within the brain. . $6,31,32^{2}$ For example, Angelucci et $\mathrm{al}^{31}$ demonstrated that cognitive training elevates BDNF in individuals with PD. The mechanism by which BDNF improves cognition is not yet fully understood, nor is it likely to be the sole mechanism responsible for clinically significant change, but only one of the components of which we are currently aware. While cognitive stimulation differs from cognitive training, the former therapy involving discussion and activities as opposed to guided practice on a standard set of cognitive tasks, ${ }^{33}$ an association between cognitive stimulation and serum BDNF has also been reported. ${ }^{26}$ The latter study is of particular relevance to the current research as the CST was delivered by family members in the home environment. Collectively, these studies suggest that if cognitive stimulation has the potential to elevate BDNF, the growth factor could be considered for use as a biomarker in future large-scale clinical trials of CST and adapted versions.

In addition to neurobiological variables, it is important to consider psychological and social factors. Social stigma or other socially imposed barriers can be a significant source of distress and thus impact on outcome. ${ }^{34}$ Examples of psychosocial issues include negative social interactions, unemployment or early retirement due to symptoms, physical disability that prevents access to certain environments and changes to family life, such as increased dependency on a companion or partner. ${ }^{34-37}$ Negative psychosocial factors can lead to a decrease in personal efficacy, psychological well-being, and relationship satisfaction and increased social isolation and depression. ${ }^{35,36,38,39}$ CST-PD will seek to address the negative psychosocial outcomes of PDD, PD-MCI and DLB by providing opportunities for companion-supported mastery experiences; having the therapy delivered by a known and significant other may allow people with PRD to feel more comfortable challenging themselves in cognitively stimulating activities. Improvements in perceived personal efficacy, through mastery experiences, could foster interest and (re)engagement in social or previously enjoyed activities, lower vulnerability to depression and improve relationship satisfaction. $^{40}$

It is also important to consider personal efficacy from the companions' point of view: the change effected by the intervention may be influenced by the companions' beliefs in their ability to deliver the therapy (instructional efficacy ${ }^{39}$ ). Companions' level of perceived efficacy to motivate the people with PRD and promote mental stimulation may affect the therapy environment, the level of adherence, the quality of therapy delivered and, ultimately, outcomes for people with PRD (eg, cognition and quality of life). In school-based studies, instructors with high levels of instructional efficacy are reported to devote more time to learning, offer the appropriate level of help and praise and encourage autonomy. ${ }^{41}$ While CST-PD does not aim to create a school-based environment at home, there is potential for companions' instructional efficacy to act as a change agent and influence therapy outcomes. Throughout the development of CST-PD, companions will be consulted to identify their needs and to develop the necessary recourses to support instructional efficacy.

\section{Stage 2 - design (modeling and piloting phases)}

In the initial meeting with stakeholders (clinical experts, researchers and a PPI advisory group), user consultations took place to identify issues related to the nature of tasks and the therapy environment. Three key factors were identified: 1) the potential impact of symptom-specific issues on the therapy, for example, physical limitations from motor impairments, fluctuating levels of alertness and motor function, hallucinations and/or delusions, person-specific cognitive impairment, high levels of apathy and ability to promote self-management; 2) companions' confidence in delivering CST-PD; and 3) whether physical activity-based tasks would be appropriate as part of the therapy. To fully address these issues, perspectives from professionals, companions and people with PRD were sought.

\section{Methods}

\section{Participants}

PD professionals $(n=5)$, patient participants $(n=3)$ and companions $(n=2)$ were recruited from neurology and geriatric psychiatry services in Greater Manchester. Patient participants had a diagnosis of PD-MCI, PDD or DLB according to the appropriate consensus criteria for each condition. ${ }^{42-46}$

\section{Procedure}

The study received ethical approval from Yorkshire and the Humber-Bradford Leeds Research Ethics Committee (Reference: 15/YH/0024). All participants provided written informed consent or had a nominated consultee who provided the appropriate declaration. Focus groups were conducted by a CST-trained cognitive neuropsychologist and a psychology research assistant at the University of Manchester. PD professionals, companions (in this case, spouses) and people with 
PRD were invited to attend separate focus groups. Based on the methods by Krueger and Casey ${ }^{47}$ and adapted for dementia by Bamford and Bruce, ${ }^{48}$ the groups were intentionally small, with no more than eight participants plus facilitators. As some companions and people with PRD were unable to attend the focus groups due to unforeseen medical or caring responsibilities, a second focus group was held for companions and semi-structured interviews were conducted with a further companion and two people with PRD, in the home setting.

A semi-structured interview design probed opinions relating to a number of issues specific to people with PD and DLB, as well as those identified by our PPI advisory group, including the suitability of tasks to motor difficulties, psychiatric issues and cognitive difficulties. Digital voice recordings were made of all focus groups and interviews to enable transcription and analyses.

\section{Focus groups}

Professionals were split into two groups and asked to evaluate six activities, spanning three topics, plus evaluate the introduction section of the original iCST manual. The groups were then asked to describe their evaluation to the other party, before having an open discussion of the points identified by the PPI advisory group. Finally, opinion was sought for five new topics, devised by the research team, before concluding the focus group with a yes/no topic inclusion/exclusion checklist to determine which subject areas were deemed most appropriate for the adapted therapy.

In the focus group for companions, participants were invited to try several of the activities, in pairs, over a range of different topic areas. Open discussion took place after each topic, with focus on the issues identified by the PPI advisory group. The focus group concluded with a yes/no topic inclusion/exclusion checklist.

The focus group of people with PRD involved a short group CST session and preferences were observed from the conversations that resulted. This focus group also concluded with a yes/no topic inclusion/exclusion checklist.

\section{Transcript analysis and reporting}

To ensure an iterative development process, data analysis ran alongside data collection, that is, subsequent focus groups were adapted using information obtained from previous sessions. The process of analysis began with reading and re-reading each transcript. Salient comments were noted and grouped by commonality, within and across transcripts. Rereading, comparison and re-grouping of comments continued until the main themes and subthemes were identified. Data were reported in accordance with an abbreviated consolidated criteria for reporting qualitative research (COREQ) structure. ${ }^{49}$

\section{Results}

Three main themes were identified and are presented in Table 1 with subthemes and frequency of comments. The professionals provided comments on all but 3 of the 28 subthemes (89\%). In contrast, companions and patients identified with a substantial proportion of the subthemes ( $64 \%$ and $61 \%$, respectively), but tended to focus on specific issues that they considered most relevant to them.

To accurately represent the views of the participants, a single quote from each theme has been selected (agreed among authors), with five additional examples in Table 2.

\section{Theme I: manual form and content}

All parties agreed that the content needed updating (eg, add topics on technology, computers, mobile phones) as well as making topics culturally inclusive. Professionals suggested that the topics should include: art, history, geography, pets, books, transport and sports (eg, golf and cycling), among others. People with PRD did not want to avoid sensitive topics, but rather viewed CST-PD as an opportunity to facilitate discussion that otherwise would not usually happen:

[...] would it be possible for people like myself to have a general discussion on "do you miss work?", "what elements of work do you miss?". I can't see it will work for all but maybe for some, you know [...] I miss work so much, I miss the hassle, I miss the estimating, I miss the hiring, the firing, doing wages and all sorts. If I hadn't got ill, I don't think I would have ever retired. [Person with PRD]

In the iCST manual, each topic is split into two levels of difficulty; however, professionals highlighted that this format may be demotivating for those with deteriorating level of cognitive function. Professionals also felt that color-coded sections would be of benefit to ease the selection of topics based on one's interest. There was consensus that lengthy paragraphs should be avoided and more images should be included to keep topics from becoming dry and cluttered. Companions also suggested the use of good-quality and thin paper, as thicker paper can cause problems with page turning in people with tremor. Professionals and companions also pointed out that any speech impairment might make reading aloud difficult, so any instructions to read aloud should be optional. 
Table I Summary of themes and subthemes identified from focus groups and interviews

\begin{tabular}{|c|c|c|c|c|}
\hline \multirow[t]{2}{*}{ Themes and subthemes } & \multicolumn{3}{|c|}{ Frequency of comments } & \multirow[t]{2}{*}{ Total } \\
\hline & Professionals & Companions & People with PRD & \\
\hline \multicolumn{5}{|l|}{ Manual form and content } \\
\hline Topic variety and development & 8 & 4 & 21 & 33 \\
\hline Modernization & 8 & 6 & 1 & 15 \\
\hline Expanding on previous interests & 2 & 7 & I & 10 \\
\hline Bringing the outside in & 3 & 2 & 3 & 8 \\
\hline Difficulty & 3 & 1 & 4 & 8 \\
\hline Cultural relevance & 7 & - & - & 7 \\
\hline Formatting & 3 & 1 & 1 & 5 \\
\hline Quality & 2 & - & 3 & 5 \\
\hline \multicolumn{5}{|l|}{ Therapy acceptability in PD } \\
\hline Hallucinations and perceptual issues & 16 & - & 2 & 18 \\
\hline Motor issues & 12 & 4 & - & 16 \\
\hline General suitability & - & - & 12 & 12 \\
\hline Cognition & 1 & 3 & 4 & 8 \\
\hline Eating (anosmia, swallowing, salivation, dexterity) & 6 & - & - & 6 \\
\hline Apathy & - & 4 & 2 & 6 \\
\hline Location of therapy & I & 2 & 1 & 4 \\
\hline Delusions & 4 & - & - & 4 \\
\hline Punding & I & 3 & - & 4 \\
\hline Isolation & I & - & 1 & 2 \\
\hline Speech difficulties & I & - & - & 1 \\
\hline Impulse control (finances) & I & - & - & I \\
\hline Anxiety & - & I & - & 1 \\
\hline \multicolumn{5}{|l|}{ Companion considerations } \\
\hline Improving written guidance for companions & 7 & 3 & 1 & 11 \\
\hline Therapy acceptability for companions & 2 & 6 & 1 & 9 \\
\hline Involving others & 2 & 3 & I & 6 \\
\hline Avoid confrontation & I & 2 & 2 & 5 \\
\hline Training & 2 & - & 2 & 4 \\
\hline Failures and successes & 2 & 1 & - & 3 \\
\hline Planning in advance & I & I & - & 2 \\
\hline
\end{tabular}

Abbreviations: PD, Parkinson's disease; PRD, Parkinson's-related dementia.

\section{Key recommendations}

- Encourage opinion-based questions.

- Include more photography, limit paragraph length.

- Modernize and extend the topic content, make it multicultural.

- Remove the two discrete levels of complexity and gradually increase the complexity of the tasks within each topic.

- Color code topic sections.

- Include optional instructions to read information aloud.

\section{Theme 2: therapy acceptability in PD}

All parties expressed the desire for the CST-PD to improve cognition, and professionals suggested card games as a potential tool to help with strategy and memory. Companions felt that the inclusion of constructive activities might successfully distract people with PRD from the compulsive, repetitive and stereotyped behaviors known as punding. Companions and people with PRD expressed polarized views of apathy: companions were concerned about getting individuals started with the therapy, whereas people with PRD were more positive and felt that the therapy would be motivating, encouraging and would alleviate boredom:

I love things like this, I do. I enjoy it. It's good fun as well and it gives you something to talk about. [Person with PRD]

Professionals and people with PRD commented on visual and perceptual issues and pointed out that in the original iCST manual, some images were too busy and confusing with many shapes and colors that may cause hallucinations. The professionals were keen for art topics to remain, but wanted art images to be less vivid, disturbing and chaotic. Professionals also thought that abstract questions relating to art (eg,"Do you think there is a message behind the painting?") or superstition-based questions should be reconsidered and revised to avoid precipitating or perpetuating complications such as delusions. 
Table 2 Additional examples of content within each major qualitative theme

\begin{tabular}{|c|c|}
\hline Themes & Example quote \\
\hline \multirow{9}{*}{$\begin{array}{l}\text { Manual form and } \\
\text { content }\end{array}$} & "I think it is not fit for today's purposing. There is no mention of computers, mobile phones..." (companion) \\
\hline & "It's important to make it multi-cultural but keep it British”; “(include) the world, the local, the personal” (professional) \\
\hline & "Focus on things that they once used to enjoy doing that they are not doing any longer. It is quite possible they could do it \\
\hline & in a different way" (professional) \\
\hline & "And maybe color guide. You know how sometimes in books you have sections that are colored off and you can just turn \\
\hline & to them". [The person with PRD might say] "that pink section was good last time and we'll go back to the pink section" \\
\hline & (professional) \\
\hline & "It might be best not to include the difficulty levels cause you can almost see someone doing this again a year later and \\
\hline & finding out they can't use level B anymore, whereas they did last year and it kind of emphasises deterioration" (professional) \\
\hline \multirow{12}{*}{$\begin{array}{l}\text { Therapy acceptability } \\
\text { in PD }\end{array}$} & "It's good to be able to discuss. I mean, you are reminding me of things and that's encouraging me, that's picking me up a \\
\hline & bit” (person with PRD) \\
\hline & "Cause it helps you, to keep your memory and things. If you are using that part of your brain on a regular basis, I can see it \\
\hline & being helpful” (person with PRD) \\
\hline & "It's very easy to stay at home but sometimes you just wake up and you think oh, what am I going to do today and it's that \\
\hline & passing time... I want to do something that I am interested in and I think that's something that holds us all" (person with \\
\hline & PRD interview) \\
\hline & "I think if you have someone who is tremor dominant, I would be concerned about what happened if they failed (physical \\
\hline & activity). So it's about symptoms rather than activity... (It is) probably best not to have these things in" (professional) \\
\hline & "We were concerned about the hallucinogenic nature of the images. Not very calming. We quite liked the idea of discussing \\
\hline & paintings but you would have to be careful with the images. Quite a lot of these images can freak out Parkinson's patients" \\
\hline & (professional) \\
\hline \multirow{8}{*}{$\begin{array}{l}\text { Companion } \\
\text { considerations }\end{array}$} & "It depends on your relationship at that moment and whether you've had a good week or a bad week. And what pressures \\
\hline & you've got as a carer... It is not always practical and sensible to sit down because things can get volatile" (companion) \\
\hline & "You've got to have absolute patience... It's very difficult to understand just how miserable a person can be in the wrong \\
\hline & hands" (person with PRD interview) \\
\hline & "I am not saying I could keep it going for twenty minutes necessarily" (companion) \\
\hline & "Don't forget, if people are carers and perhaps not sleeping, they are just so, so tired. They won't even get through a page \\
\hline & of this (introduction) text" (professional) \\
\hline & "It surely got to be a shared effort between the therapist" (person with PRD interview) \\
\hline
\end{tabular}

Abbreviations: PD, Parkinson's disease; PRD, Parkinson's-related dementia.

With regards to motor impairment, all participants agreed that delivering CST-PD in the home was most acceptable, and might help reduce feelings of isolation or boredom. Professionals and some companions were concerned about the physical activity-based tasks in the existing therapy. Following a lengthy discussion, it was determined that without individual physiotherapist assessment, the potential risks of including physical exercise activities outweighed the potential gain, and therefore, it was recommended that dedicated physical exercise be excluded from the activities in CST-PD.

\section{Key recommendations}

- Include card/strategy games and constructive activities.

- Exclude physical activity tasks.

- Avoid disturbing images and abstract questions.

\section{Theme 3: companion considerations}

All parties were cautious when discussing the acceptability of the therapy for companions with a caregiving role. Professionals and companions acknowledged that dyads engaging in constructive discussion might be of benefit, but exhaustion and feelings of pressure of companions and family members could not be disregarded. Involving other family members (eg, grandchildren) was suggested in all focus groups. Participants felt that involving family members would be a pleasurable experience and would limit any burden that a dyadic therapy might introduce:

We are together all the time. Sometimes the relationship gets strained because you are at the end of it. So, I think my daughter could get my husband to do a lot more than I could. She could sit down with him and talk to him about things that he just says to me "Oh you are not listening". So I think it is imperative to involve your immediate family. (Companion)

Companions communicated concerns about self-doubt and uncertainty in delivering the therapy, and all participants agreed advice should be provided on what to do in situations of confrontation. With respect to the current iCST manual, all participants felt that the introduction was too long and overly complicated, and suggested reducing it to a single 
page with 10 key points including a summary of what is in the manual, what support there is and how to find it. The importance of appropriate training was of interest to professionals and people with PRD, with professionals suggesting that companions read through materials in advance of the therapy session.

\section{Key recommendations}

- Involve others (family members, grandchildren, friends).

- Streamline the introduction.

- Provide advice on managing challenging behavior.

- Include strategies to build instructional efficacy.

\section{Stage 3: test and refine}

At the end of the design stage, a final draft CST-PD therapy manual was produced. This was subsequently field tested by dyads ie, people with PRD and companions (Part A). The purpose was to evaluate the therapy manual (in context) against the user requirements defined in Stage 2. Additional opinion was also sought from PPI representatives at a local Parkinson's UK branch meeting to better understand if the intervention and delivery processes could work in a real world (Part B). ${ }^{50}$

\section{Part A (companions and people with PRD) Methods}

Participants

Companions $(n=3)$ and people with PRD $(n=3)$ participated in the field testing. Participants were recruited from local neurology and geriatric psychiatry services in Greater Manchester and had a diagnosis of either PD-MCI, PDD or DLB according to the appropriate consensus criteria for each condition. ${ }^{42-46}$

\section{Procedure}

The study received favorable ethical opinion from Yorkshire and the Humber-Bradford Leeds Research Ethics Committee (Reference: 15/YH/0531). Dyads completed a portion ( 2 weeks; $\sim 6$ sessions) of the program with support from the research team. At the end of the 2-week period, dyads completed a usability assessment and provided detailed qualitative feedback. The usability assessment was based on the 10-item System Usability Scale, ${ }^{51,52}$ which was adapted to suit the needs of this study and population (ie, the term "system" was replaced with "therapy manual" and additional questions relating to formatting, layout and so on were included). The adapted assessment consisted of 24 statements (eg, "I found the manual unnecessarily complex" and "I would imagine that most companions would learn to use the manual very quickly") to which the participants provided free answers. Items were categorized with relevance to themes from the focus groups and interviews, and are discussed accordingly.

\section{Results}

Of the 24 items, 11 statements were grouped under Theme 1, "Manual form and content", 9 under Theme 2, "Therapy acceptability in PD", and 4 under Theme 3, "Companion considerations".

\section{Theme I: manual form and content}

All participants found the manual layout was consistent, the pages were easy to navigate, the topics were well-integrated with plenty of choice, and the size and color of the print was good. Participants reported they would appreciate additional materials consisting of games, adult coloring and stationary.

\section{Theme 2: therapy acceptability in PD}

All participants with PRD agreed that they would feel confident using the manual and would likely use it three times per week. The task complexity was perceived differently depending on the level of cognitive impairment; the participant with PD-MCI found the level of complexity appropriate, and the participant with PDD found the tasks challenging. The person with DLB found the manual, images and tasks challenging. The participant with DLB and their companion agreed that the manual was not suitable, with the companion attributing this to the advanced level of cognitive impairment of the participant.

\section{Theme 3: companion considerations}

Both participants with dementia, but not the participant with PD-MCI, were definitive that they would need support to use the manual. All companions agreed that they would be able to learn to use the manual quickly, although they felt it beneficial to have some preparation time before commencing use of the manual.

\section{Key recommendations}

- Companion-training package should include preparation time.

- Additional support may be required depending on the level of cognitive impairment. 


\section{Part B (PPI representatives)}

\section{Methods}

Participants

Thirty-three PPI representatives (companions and people with PRD) from a local Parkinson's UK community group took part.

\section{Procedure}

PPI representatives piloted the therapy questions and topic guides in a single group session held at a Parkinson's UK support group. Attendees were given the option of completing a feedback form to comment on the usability and acceptability of the therapy. Four topics were randomly selected from the manual for use on the day: herbs, newspapers, Blackpool and ball games. A modified version of the in-house usability assessment, using a five-point Likert response scale ( $1=$ "strongly disagree" to $5=$ "strongly agree"), with one question reverse-scored, was circulated to gather feedback.

This phase of the study was carried out as a PPI session and, as such, ethical approval was not obtained to record opinion from the PD UK community group. Feedback was gathered anonymously and no further information (identifiable or otherwise) was collected about the volunteers.

\section{Results}

Sixteen volunteers elected to complete the feedback form. Responders strongly agreed that it was easy to engage in the session (median $=5$ ), to stimulate conversation using the topic materials (median $=5$ ), and that the discussion was stimulating and interesting (median $=5$ ). Responders also agreed that the session content (median $=4.5$ ) and images (median $=4$ ) were clear and easy to understand, that there was opportunity to personalize the session (median $=4$ ) and that they would like to do a similar activity in the future (median $=4$ ). Volunteers were uncertain about whether the level of complexity was suitable for someone with memory problems (median $=3$, "neither agree nor disagree").

\section{Key recommendation}

In future evaluations, assess topic acceptability in people with moderate-severe cognitive impairment.

\section{Discussion}

This research adapted and tested a psychosocial therapy for people with PRD and their companions. A user-centered design was employed, and professionals, people with PRD, companions and researchers worked collaboratively to modify an existing therapy to meet specific user needs.
The first step was to identify and review the evidence base for psychosocial therapies for people with PRD and the potential mechanisms of change. Subsequently, a candidate therapy for adaptation was selected. A basic prototype manual (loose pages and sample topic ideas) was developed early in the second phase to allow the proposed therapy to be discussed in a more meaningful way with stakeholders. The final version of the therapy manual was developed iteratively through a series of focus groups, interviews, a group demonstration and field testing.

An advantage of co-developing the intervention with stakeholders is that it limits the risk of missing critical therapeutic requirements and provides a positive and satisfying experience for the individuals involved (ie, the therapy meets the goals of its users because its users have been actively involved in the iterative development of the therapy). This aspect was particularly important for CST-PD, as the target population is frail, the symptoms complex and a therapy companion is required. As the specific needs of this population are not well understood, the design of the therapy could not be informed through literature only. Hence, we involved people with PRD, companions and health professionals as partners throughout the design process. Testament to CST-PD meeting the needs of people with PRD and their companions is the positive feedback received from the Parkinson's UK field testing session. The comments indicated that the members engaged with the session and enjoyed the activities. Although speculative at this stage, these findings suggest the therapy might address the neuropsychiatric symptoms (eg, apathy and anxiety) associated with increased caregiver distress in PRD. ${ }^{50}$ The successful delivery of the session in a group format also highlights the versatility of the adapted therapy and indicates that it may be appropriate for people with PRD attending group-based sessions.

This study has some limitations. The number of participants is small, and focus group methodology proved challenging for this companion and patient population, given their complex and changeable needs. The obstacles faced in bringing participants together for a focus group demonstrate how difficult it might be for companions and people with PRD to attend group CST sessions, and reinforce the appropriateness of developing a home-based therapy that can be carried out at the convenience of the people involved.

\section{Conclusion}

There is an urgent need to expand the management options for people with PRD beyond standard pharmacologic approaches. Psychosocial therapies suitable for this group 
of people may, therefore, have a vital role. The development phase of CST-PD was extensive and involved an iterative process comprising theory-driven recommendations, evaluation of users' needs, prototype manual development and field testing. This aspect of the trial resulted in the production of CST-PD, a therapy manual specifically tailored for people with PRD and their companions. Following the MRC guidelines, ${ }^{9}$ a pilot randomized clinical trial is currently underway to examine the feasibility, acceptability and tolerability of CST-PD and to explore the outcome measures. ${ }^{53}$ The pilot data will be used to inform a subsequent, definitive, multisite randomized controlled trial.

\section{Acknowledgments}

This article presents independent research supported and funded by the National Institute for Health Research, under the Research for Patient Benefit (RfPB) Program (competition number 22; grant number PB-PG-0613-31058). The views expressed in this article are those of the authors and not necessarily those of the NHS, the NIHR or the Department of Health.

The authors would like to acknowledge and thank the original CST and iCST development team for permitting the adaptation of their therapy, the R\&D personnel involved in study set-up and maintenance, and the NHS clinicians and nursing teams involved in referral of participants to the study. The authors would also like to thank the PPI representatives for their helpful advice and continued support at various stages of the project. The authors kindly acknowledge the contribution of Professor Martin Orrell, Dr Monty Silverdale and $\mathrm{Dr}$ Bo Fu in the conception and design of CST-PD.

\section{Authors' contributions}

SAM and KRM prepared the manuscript. KRM and SV recruited participants, facilitated focus groups, conducted individual interviews and conducted the analysis of the data gathered. IL, VO, KRM, SAM, SV, EP and SS were involved in the conception and design of the CST-PD approach, the critical appraisal of the intellectual content, manuscript revisions and final approval prior to publication.

\section{Disclosure}

The authors report no conflicts of interest in this work.

\section{References}

1. The British Psychological Society and The Royal College of Psychiatrists. Dementia: A NICE-SCIE Guideline on supporting people with dementia and their carers in health and social care. 1st ed. London: Aldren Press; 2007.
2. Aarsland D, Larsen JP, Lim NG, et al. Range of neuropsychiatric disturbances in patients with Parkinson's disease. J Neurol Neurosurg Psychiatry. 1999;67(4):492-496.

3. Witjas T, Kaphan E, Azulay JP, et al. Nonmotor fluctuations in Parkinson's disease: frequent and disabling. Neurology. 2002;59(3): 408-413.

4. Cahn DA, Sullivan EV, Shear PK, Pfefferbaum A, Heit G, Silverberg G. Differential contributions of cognitive and motor component processes to physical and instrumental activities of daily living in Parkinson's disease. ArchClin Neuropsychol. 1998;13(7):575-583.

5. Pont-Sunyer C, Hotter A, Gaig C, et al. The onset of nonmotor symptoms in Parkinson's disease (The ONSET PD Study). Mov Disord. 2015;30(2):229-237.

6. Aarsland D, Bronnick K, Williams-Gray C, et al. Mild cognitive impairment in Parkinson disease. A multicenter pooled analysis. Neurology. 2010;75(12):1062-1069.

7. Hely MA, Reid WG, Adena MA, Halliday GM, Morris JG. The sydney multicenter study of Parkinson's disease: the inevitability of dementia at 20 years. Mov Disord. 2008;23(6):837-844.

8. Ballard CG, Aarsland D, McKeith I, et al. Fluctuations in attention: PD dementia vs DLB with parkinsonism. Neurology. 2002;59(11): 1714-1720.

9. Craig P, Dieppe P, Macintyre S, Michie S, Nazareth I, Petticrew M; Medical Research Council Guidance. Developing and evaluating complex interventions: the new Medical Research Council guidance. BMJ. 2008;337:a1655.

10. Orgeta V, McDonald KR, Poliakoff E, Hindle J, Clare L, Leroi I. Cognitive training interventions for dementia and mild cognitive impairment in Parkinson's disease. Cochran Database Syst Rev. 2015;11: Art. No. CD011961.

11. Goransson B. The re-design of a PDA-based system for supporting people with Parkinson's disease. In: Fincher S, Panos M, Moore D, Ruddle R, eds. People and Computers XVIII - Design for Life: Proceedings of HCI 2004. London: Springer; 2005:181-196.

12. Mays N, Roberts E, Popay J. Synthesizing research evidence. In: Fulop N, Allen P, Clarke A, Black N, eds. Studying the Organization and Delivery of Health Services: Research Methods. London: Routledge; 2001:188-220.

13. Taulbee LR, Folsom JC. Reality orientation for geriatric patients. Hosp Community Psychiatry. 1966;17(5):133-135.

14. Norris A. Reminiscence With Elderly People. Bicester: Winslow Press; 1986.

15. Butler RN. The life review: an interpretation of reminiscence in the aged. Psychiatry. 1963;26(1):65-76.

16. Woods B, Aguirre E, Spector AE, Orrell M. Cognitive stimulation to improve cognitive functioning in people with dementia. Cochrane Database Syst Rev. 2012;2:CD005562.

17. Woods RT, Orrell M, Bruce E, et al. REMCARE: pragmatic multicenterrandomizedtrial of reminiscence groups for people with dementia and their family carers: effectiveness and economic analysis. PLoS One. 2016;11(4):e0152843.

18. Guidelines APA. Practice guideline for the treatment of patients with alzheimer's disease and other dementias of late life. American Psychiatric Association. Am J Psychiatry. 1997;154(5 Suppl):1-39.

19. Spector A, Thorgrimsen L, Woods B, et al. Efficacy of an evidencebased cognitive stimulation therapy program for people with dementia. Br J Psychiatry. 2003;183:248-254.

20. Knapp M, Thorgrimsen L, Patel A, et al. Cognitive stimulation therapy for people with dementia: cost-effectiveness analysis. Br J Psychiatry. 2006;188(6):574-580.

21. Orrell M, Aguirre E, Spector A, et al. Maintenance cognitive stimulation therapy for dementia: single-blind, multicentre, pragmatic randomized controlled trial. Br J Psychiatry. 2014;204(6):454-461.

22. Orrell M, Spector A, Thorgrimsen L, Woods B. A pilot study examining the effectiveness of maintenance cognitive stimulation therapy (MCST) for people with dementia. Int J Geriatr Psychiatry. 2005;20(5): 446-451. 
23. Leung P, Yates L, Orgeta V, Hamidi F, Orrell M. The experiences of people with dementia and their carers participating in individual cognitive stimulation therapy. Int J Geriatr Psychiatry. Epub 2017 Feb 20.

24. Orrell M, Yates L, Leung P, et al. The impact of individual cognitive stimulation therapy (iCST) on cognition, quality of life, caregiver health, and family relationships in dementia: a randomized controlled trial. PLoS Med. 2017;14(3):e1002269.

25. Leroi I, McDonald K, Pantula H, Harbishettar V. Cognitive impairment in Parkinson disease: impact on quality of life, disability, and caregiver burden. J Geriatr Psychiatry Neurol. 2012;25(4):208-214.

26. Farzana F, Sreekanth V, Mohiuddin MK, Mohan V, Balakrishna N, Ahuja YR. Can individual home-based cognitive stimulation therapy benefit Parkinson's patients with mild to moderate cognitive impairment? Int J Geriatr Psychiatry. 2015;30(4):433-435.

27. Nasreddine ZS, Phillips NA, Bédirian V, et al. The montreal cognitive assessment, MoCA: a brief screening tool for mild cognitive impairment. J Am Geriatr Soc. 2005;53(4):695-699.

28. Jenkinson C, Fitzpatrick R, Peto V, Greenhall R, Hyman N. The Parkinson's diisease questionnaire (PDQ-39): development and validation of a Parkinson's disease summary index score. Age Ageing. 1997; 26(5):353-357.

29. Thorgrimsen L, Selwood A, Spector A, et al. Whose quality of life is it anyway?: the validity and reliability of the quality of life-alzheimer's disease (QoL-AD) scale. Alzheimer Dis Assoc Disord. 2003;17(4): 201-208.

30. Craig P, Dieppe P, Macintyre S, Michie S, Nazareth I, Petticrew M. Developing and evaluating complex interventions: The new Medical Research Council guidance. Int J Nurs Stud. 2013;50(5):587-592.

31. Angelucci F, Peppe A, Carlesimo GA, et al. A pilot study on the effect of cognitive training on BDNF serum levels in individuals with Parkinson's disease. Front Hum Neurosci. 2015;9:130.

32. Farzana F, Ahuja YR, Sreekanth V. Non-pharmacological interventions for enhancing brain plasticity and promoting brain recovery: a review. Res Neurosci. 2013;2(3):39-49.

33. Clare L, Woods RT. Cognitive training and cognitive rehabilitation for people with early-stage alzheimer's disease: a review. Neuropsychol Rehabil. 2004;14(4):385-401.

34. Simpson J, McMillan H, Reeve D. Reformulating psychological difficulties in people with Parkinson's disease: the potential of a social relational approach to disablism. Parkinsons Dis. 2013;2013:608562.

35. Reeve D. Towards a psychology of disability:the emotional effects of living in a disabled society. In: Goodley D, Lawthorn R, eds. Disability and Psychology: Critical Introductions and Reflections. Basingstoke, UK: Macmillan; 2006:94-107.

36. Schrag A, Hovris A, Morley D, Quinn N, Jahanshahi M. Young-versus older-onset Parkinson's disease: impact of disease and psychosocial consequences. Mov Disord. 2003;18(11):1250-1256.

37. Joachim $\mathrm{G}$, Acorn S. Stigma of visible and invisible chronic conditions. J Adv Nurs. 2000;32(1):243-248.
38. Sindhi A, Leroi I. Nonpharmacological therapies for cognitive enhancement in Parkinson's disease: applying old interventions in a new setting? Neurodegener Dis Manag. 2013;3(6):539-547.

39. Brown R, Jahanshahi M. Depression in Parkinson's disease: a psychosocial viewpoint. Adv Neurol. 1995;65:61-84.

40. Bandura A. Perceived self-efficacy in cognitive development and functioning. Educ Psychol. 1993;28(2):117-148.

41. Gibson S, Dembo MH. Teacher efficacy: a construct validation. JEduc Psychol. 1984;76(4):569.

42. Hughes AJ, Daniel SE, Kilford L, Lees AJ. Accuracy of clinical diagnosis of idiopathic Parkinson's disease: a clinico-pathological study of 100 cases. J Neurol Neurosurg Psychiatry. 1992;55(3):181-184.

43. Emre M, Aarsland D, Brown R, et al. Clinical diagnostic criteria for dementia associated with Parkinson's disease. Mov Disord. 2007; 22(12):1689-1707; quiz 1837.

44. Dubois B, Burn D, Goetz C, et al. Diagnostic procedures for Parkinson's disease dementia: recommendations from the movement disorder society task force. Mov Disord. 2007;22(16):2314-2324

45. McKeith IG, Dickson DW, Lowe J, et al. Diagnosis and management of dementia with lewy bodies - third report of the DLB consortium. Neurology. 2005;65(12):1863-1872.

46. Litvan I, Goldman JG, Troster AI, et al. Diagnostic criteria for mild cognitive impairment in Parkinson's disease: Movement Disorder Society Task Force guidelines. Mov Disord. 2012;27(3):349-356.

47. Krueger RA, Casey MA. Focus Groups:A Practical Guide for Applied Research. 4th ed. London: SAGE; 2009.

48. Bamford C, Bruce E. Successes and challenges in using focus groups with older people with dementia. In: Wilkinson $\mathrm{H}$, ed. The Perspectives of People With Dementia:Research Methods and Motivations. London: Jessica Kingsley; 2002:139-164.

49. Tong A, Sainsbury P, Craig J. Consolidated criteria for reporting qualitative research (COREQ): a 32-item checklist for interviews and focus groups. Int J Qual Health Care. 2007;19(6):349-357.

50. O'Cathain A, Thomas KJ, Drabble SJ, Rudolph A, Goode J, Hewison J. Maximizing the value of combining qualitative research and randomized controlled trials in health research: the QUAlitative Research in Trials (QUART) studya mixed methods study. Health Technol Assess. 2014;18(38):1-197, v-vi.

51. Sauro J. Measuring Usability With The System Usability Scale (SUS). 2011. Available from: http://www.measuringu.com/sus.php. Accessed February 1, 2016.

52. Brooke J. SUS-A quick and dirty usability scale. Usability Evaluation in Industry. 1996;189(194):4-7.

53. McCormick SA, McDonald KR, Vatter S, et al. Psychosocial therapy for Parkinson's-related dementia: study protocol for the INVEST randomised controlled trial. BMJ Open. 2017;7(6):e016801.
Clinical Interventions in Aging

\section{Publish your work in this journal}

Clinical Interventions in Aging is an international, peer-reviewed journal focusing on evidence-based reports on the value or lack thereof of treatments intended to prevent or delay the onset of maladaptive correlates of aging in human beings. This journal is indexed on PubMed Central, MedLine,

\section{Dovepress}

CAS, Scopus and the Elsevier Bibliographic databases. The manuscript management system is completely online and includes a very quick and fair peer-review system, which is all easy to use. Visit http://www.dovepress. com/testimonials.php to read real quotes from published authors. 